\title{
SOLUTION OF YOUNG-LAPLACE EQUATION WITH FINITE-VOLUME METHOD AND OVERLAPPED GRID
}

\author{
ZEYI ZHANG ${ }^{1,2}$ \& LIQIU WANG ${ }^{1,2}$ \\ ${ }^{1}$ Department of Mechanical Engineering, the University of Hong Kong, Hong Kong. \\ ${ }^{2} \mathrm{HKU}$-Zhejiang Institute of Research and Innovation (HKU-ZIRI), China.
}

\begin{abstract}
The Young-Laplace equation describes the stress balance between the interfacial tension and the gravitational body force. Its modified form can be applied to model the dynamics of the interface of the two-phase flow. It is analytically difficult to solve the Young-Laplace equation due to its strong nonlinearity, in the form of the surface curvature and the implicit body force on the interface. This work aims to numerically solve the Young-Laplace equation with a finite-volume method (FVM) and an overlapped grid. The overlapped grid allocates the variable and its derivative together at every grid point, and is compared with the traditional staggered grid. The proposed overlapped grid can achieve fourth-order numerical accuracy, which is higher than the second-order accuracy of the staggered grid. Also, the overlapped grid, with full states defined at every grid point, offers convenience to the implementation of the boundary condition as well as the coupling of multi-physics.

Keywords: finite-volume method, overlapped grid, young-laplace equation
\end{abstract}

\section{INTRODUCTION}

Two immiscible fluids form the interface $[1,2]$. The shape of the interface is influenced by the interfacial tension and the pressure difference [3, 4]. The interfacial tension aims to minimize the surface area and the surface energy [5]. The effect of the interfacial tension becomes more significant in a small length scale, measured by the Bond number (Bo) or the surface/ volume ratio [6], and the interface science is one of the key topics in fields such as microelectromechanical systems (MEMS) [7] and microfluidics [8].

Different from solid boundaries, the fluid interface is free to deform, and its shape function is particularly important as a boundary condition to be determined. The interface shape or the surface curvature is coupled with the surrounding physical processes, such as flow convection, gravity, electric field, elasticity and so on. In [9], Weber and Shandas simulated the microbubble formation as well as the two-phase flow with the interface tracking method. Dadvand et al. [9] demonstrated a numerical analysis of the drop-on-demand problem controlled by spark with the boundary element analysis, assuming small surface/volume ratio. Lim et al. [10] numerically solved the cone-jet formation under the electric field with both the front tracking and the finite-volume method (FVM). Multiple equilibria were derived by Taroni and Vella [7] for a elastocapillary system by the lubrication theory. The finite element method was adopted by Sprittles and Shikhmurzaev [11,12] to model the dynamic wetting flows and the dynamic contact angle. Moreover, the surface tension can lead to hydrodynamic instability $[13,14]$.

The Young-Laplace equation is one of the simplest models $[15,16]$ to describe the static surface shape governed by the gravitational body force and the restorative interfacial tension. One popular application is the axisymmetric drop shape analysis (ADSA) [17, 18]. Basically, ADSA compares and fits the drop shape with the numerical solutions of the Young-Laplace equation to obtain the interfacial tension and the contact angle of the liquid. This technique aided by the modern photographic technique is faster and more efficient than other traditional methods. Nevertheless, researchers are investigating methods for developing a robust and 
efficient numerical algorithem to solve the Young-Laplace equation. A finite-element-based algorithm was proposed by Dingle et al. [19]. In [20], Gille et al. compared four different numerical schemes. Also, Danov et al. [21] worked on the sixth-order Taylor method with the emphasis on the accuracy, stability and computational time.

In the current work, we propose the overlapped grid to improve the FVM modelling accuracy of the Young-Laplace equation by two orders compared with the staggered grid [22, 23]. For each grid point of the overlapped grid, there are two unknown variables allocated, $h$ and $h^{\prime}$; for the staggered grid, only one unknown variable, either $h$ or $h^{\prime}$, is allocated. Literally, we overlap one more layer of grids on top of the staggered grid. Thus, the nonlinear source term can be better approximated with reduced Taylor series truncation error by the heavily packed unknown variables [24]. Consequently, smaller effort (e.g. programming and computation) leads to higher accuracy of the overlapped grid, $O\left(\Delta x^{4}\right)$, than that of the staggered grid, $O\left(\Delta x^{2}\right)$.

This paper is constructed as per the following sections. In Section 2, the FVM discretization of the Young-Laplace equation is formulated and analyzed, with respect to both the 'traditional' staggered grid and the proposed overlapped grid. Section 3 demonstrates and discusses the numerical solutions. The two FVM solutions are verified with one published solution [4], and we further investigate the performance of the overlapped grid, with the staggered grid as a beanchmark. Finally, the concluding remarks are addressed in Section 4.

\section{MATHEMATICAL MODELLING}

\subsection{Physical model}

The Young-Laplace equation governs the capillary rise of the air-liquid interface along the flat plates. We consider the steady-state shape function, $h=h(x)$, between the two vertical parallel infinite-wide plates with the separation distance of $w$, as is schematically shown in Fig. 1 [4]. On the 2-dimensional (2D) cross-sectional plane, the Young-Laplace equation can be expressed in the partial-differential-equation (PDE) form as,

$$
\rho g h=\sigma d \theta / d s, x \in[0, w],
$$

where $\rho, g$ and $\sigma$ are the fluid density $\left(\mathrm{kg} / \mathrm{m}^{3}\right)$, the gravitational acceleration $\left(\mathrm{m} / \mathrm{s}^{2}\right)$ and the interfacial tension $\left(\mathrm{J} / \mathrm{m}^{2}\right)$, respectively. $h$ is the rising height, $s$ is the length and $\theta$ is the angle $(\theta \in[-\pi / 2, \pi / 2])$ of the surface, respectively. $\rho g h$ measures the net pressure applied on the surface due to the gravitational body force, while $\sigma d \theta / d s$ measures the net interfacial tension due to the curvature along the surface.

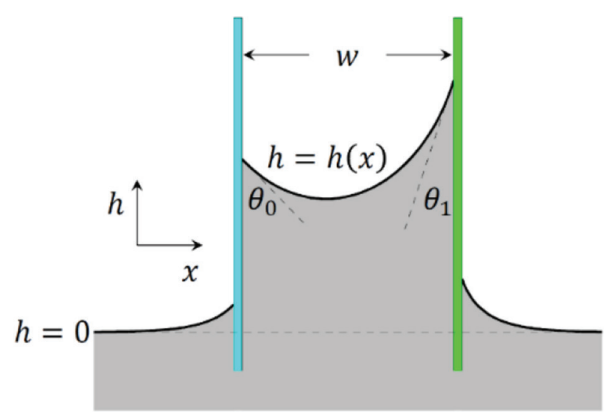

Figure 1: Schematic of the 2D free-surface capillary rise between two vertical parallel plates. 


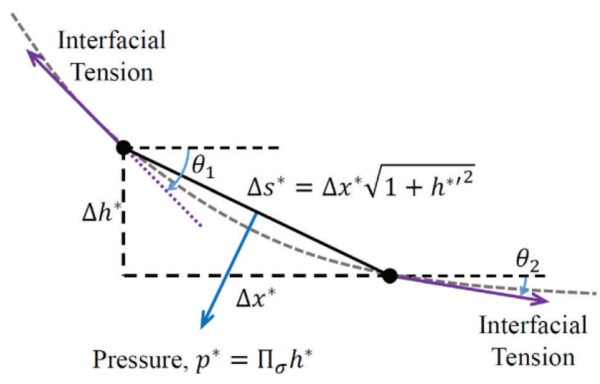

Figure 2: One finite surface with the geometry specified and unit width into the paper. The gray dashed curve represents the exact free surface, while the finite surface is the fragmental approximation of the exact solution. The interfacial tensions on the two ends have the same magnitude but different orientations, $\theta_{1}$ and $\theta_{2}$. Also, the pressure is applied on the finite surface.

The Boundary condition is in the form of the contact angles, $\theta_{0}$ and $\theta_{1}$, of the fluid on the two rigid plates,

$$
\theta(0)=\theta_{0}-\pi / 2 \text { and } \theta(w)=\pi / 2-\theta_{1} .
$$

Also, we can derive the following geometrical relationships,

$$
\tan \theta=d h / d x=h^{\prime}, \sin \theta=d h / d s, \text { and } \cos \theta=d x / d s=1 / \sqrt{1+h^{\prime 2}} .
$$

Dimensionless variables are obtained with the normalization rules,

$$
h^{*}=h / w, s^{*}=s / w, \text { and } x^{*}=x / w .
$$

And, the Young-Laplace equation can be reformed into,

$$
\Pi_{\sigma} h^{*}=d \theta / d s^{*}, x^{*} \in[0,1],
$$

where $\Pi_{\sigma}=\rho g w^{2} / \sigma$ is the dimensionless parameter, so-called Bo.

\subsection{Finite-volume method}

Equation (5) is an elliptic PDE with two boundary conditions in eqn (2). We apply the FVM to solve this nonlinear boundary-value problem. Take the line integral of eqn (5), along one finite surface from $s_{1}^{*}$ to $s_{2}^{*}=s_{1}^{*}+\Delta s^{*}$, assuming unit width into the paper, as is shown in Fig. 2 ,

$$
\int_{s_{1}^{*}}^{s_{2}^{*}} \Pi_{\sigma} h^{*} \mathrm{~d} s^{*}=\int_{s_{1}}^{s_{2}^{*}} \frac{d \theta}{d s^{*}} \mathrm{~d} s^{*},
$$

where the source term comes from the net perpendicular pressure on the finite surface, while the flux term comes from the unbalanced interfacial tension across the finite surface.

Substitute $\mathrm{d} s^{*}=\sqrt{1+h^{* 2}} \mathrm{~d} x$ and $\theta=\arctan h^{* *}$ into the above equation,

$\Pi_{\sigma} \int_{x_{1}^{*}}^{x_{2}^{*}} h^{*} \sqrt{1+h^{* \prime 2}} \mathrm{~d} x^{*}-\left(\arctan h_{2}^{* \prime}-\arctan h_{1}^{* \prime}\right)=\Pi_{\sigma} \int_{x_{1}^{*}}^{x_{2}^{*}} h^{*} \sqrt{1+h^{* \prime 2}} \mathrm{~d} x^{*}-\arctan \left(\frac{h_{2}^{* \prime}-h_{1}^{* \prime}}{1+h_{2}^{* \prime} h_{1}^{* \prime}}\right)=0$, 
(a)

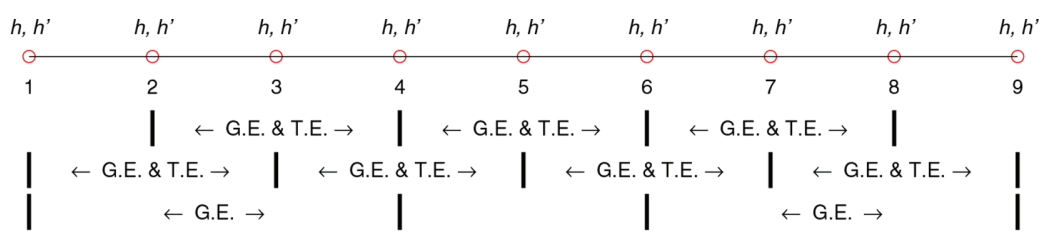

(b)

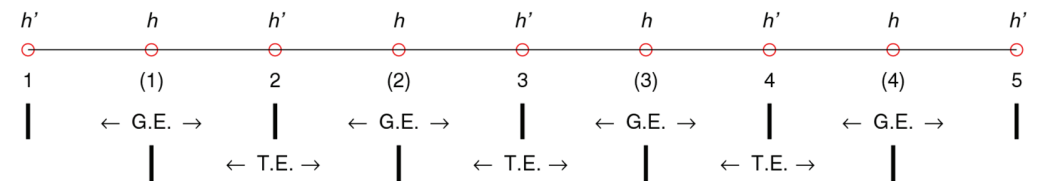

Figure 3: Two grid layouts. (a) The overlapped grid with $N_{\text {over }}=8$; (b) the staggered grid with $N_{\text {stag }}=4$. Each circle stands for a grid point, where the unknown variables ( $h$ or $h^{\prime}$ or both) are defined. 'G.E.' stands for the discretized governing equation, while 'T.E.' stands for the Taylor-expansion equation.

where the source term is $f_{s}\left(x^{*}\right)=h^{*} \sqrt{1+h^{* 2}}$, and the flux term is $f_{f}\left(x^{*}\right)=\arctan h^{* \prime}$. Thus, the Young-Laplace equation is nonlinear for the source term and the flux term, both of which depend on the unknown shape function, $h^{*}=h^{*}\left(x^{*}\right)$.

Numerically, we can further discretize eqn (7) into an algebric equation, by approximating the integral term with the unknown variables defined in the finite surface. Such approximation varies with different grid layouts. In this work, we detail two grid layouts, the "traditional" staggered grid and the proposed overlapped grid, as are shown in Fig. 3. Their main difference lies with the arrangement of the unknown variables and the algebraic equations. The staggered grid allocates the unknown variables, either $h$ or $h^{\prime}$, one by one along the free surface, while the overlapped grid allocates both $h$ and $h^{\prime}$ onto every grid point. Thus, the nonlinear source terms are approximated differently for the two grid layouts, which leads to different features of the numerical solutions. Note that the grid points are uniformly spaced in $x^{*} \in[0,1]$ for both layouts in this work.

\subsection{Staggered grid}

As is shown in Fig. 3b, the staggered grid allocates every grid point with one unknown variable, either $h$ or $h^{\prime}$. For ease of presentation, we name the finite surfaces by their centered unknown variables, such as the $h$-centered finite surface and the $h^{\prime}$-centered finite surface, respectively. The number of grids, denoted as $N_{\text {stag }}$, is counted by the number of $h$-centered finite surfaces. Thus, the total number of unknowns is $\left(2 N_{\text {stag }}+1\right)$, and the grid size is $\Delta x^{*}=1 / N_{\text {stag }}$.

The discretized governing equation is applied to the $h$-centered finite surface, while the second-order Taylor-expansion equation is applied to the complete $h^{\prime}$-centered finite surface. Moreover, the boundary condition is applied to the two $h^{\prime}$ unknowns at the two ends. In total, we can derive $\left(2 N_{\text {stag }}+1\right)$ independent algebraic equations.

\subsection{1 $\boldsymbol{h}$-centered finite surface}

Each $h$-centered finite surface is associated with three unknown variables, $h_{p}^{*}, h_{1}^{* \prime}$ and $h_{2}^{* \prime}$, where the subscript 'p' indicates the middle point between Point 1 and Point 2 . By applying the central differencing scheme, we can approximate $h_{p}^{* \prime}$ as, 


$$
h_{p}^{* \prime}=\left(h_{1}^{* \prime}+h_{2}^{* \prime}\right) / 2+O\left(\Delta x^{* 2}\right) \text {. }
$$

We use the value at the grid center to approximate the integral term in eqn (7),

$$
\int_{x_{1}^{*}}^{x_{2}^{*}} f_{s}\left(x^{*}\right) \mathrm{d} x^{*}=f_{s}\left(x_{p}^{*}\right) \Delta x^{*}+O\left(\Delta x^{* 3}\right)=h_{p}^{*} \sqrt{1+h_{p}^{* \prime 2}} \Delta x^{*}+O\left(\Delta x^{* 3}\right) .
$$

Substitute eqns (8) and (9) into eqn (7), and we can derive the discretized governing equation for the $h$-centered finite surface,

$$
\Pi_{\sigma} h_{p}^{*} \sqrt{1+\left(\frac{h_{1}^{*^{\prime}}+h_{2}^{*^{\prime}}}{2}\right)^{2}}-\frac{1}{\Delta x^{*}} \arctan \left(\frac{h_{2}^{*^{\prime}}-h_{1}^{*^{\prime}}}{1+h_{2}^{*^{*}} h_{1}^{*^{\prime \prime}}}\right)=0,
$$

which involves the three unknown variables, $h_{p}^{*}, h_{1}^{* \prime}$ and $h_{2}^{* \prime}$, and has the modelling error of $O\left(\Delta x^{* 2}\right)$.

\subsection{2 $\boldsymbol{h}^{\prime}$-centered finite surface}

Each $h^{\prime}$-centered finite surface is associated with three unknown variables, $h_{p}^{* \prime}, h_{1}^{*}$ and $h_{2}^{*}$. Their relationships are derived from the second-order Taylor expansion,

$$
\begin{aligned}
& h_{1}^{*}=h_{p}^{*}-\frac{\Delta x^{*}}{2} h_{p}^{*,}+\frac{1}{2}\left(\frac{\Delta x^{*}}{2}\right)^{2} h_{p}^{* ”}-O\left(\Delta x^{* 3}\right), \\
& h_{2}^{*}=h_{p}^{*}+\frac{\Delta x^{*}}{2} h_{p}^{* \prime}+\frac{1}{2}\left(\frac{\Delta x^{*}}{2}\right)^{2} h_{p}^{* ”}+O\left(\Delta x^{* 3}\right) .
\end{aligned}
$$

By subtracting the above two equations, we can derive the Taylor-expansion equation for the $h^{\prime}$-centered finite surface,

$$
h_{p}^{* \prime}=\left(h_{2}^{*}-h_{1}^{*}\right) / \Delta x^{*}-O\left(\Delta x^{* 2}\right), \text { or } h_{p}^{* \prime}-\left(h_{2}^{*}-h_{1}^{*}\right) / \Delta x^{*}=0,
$$

which involves the three unknown variables, $h_{p}^{*}, h_{1}^{*}$ and $h_{2}^{*}$, and has the modelling error of $O\left(\Delta x^{* 2}\right)$.

\subsection{Overlapped grid}

As is shown in Fig. 3a, the overlapped grid allocates both unknown variables, $h$ and $h^{\prime}$, to each grid point. We regard a grid consisting of three grid points as a complete finite surface, which is referred to as the three-point finite surface. Also, at the two ends of the free surface, there are two finite surfaces consisting of four grid points, and they are referred to as the fourpoint finite surface. The number of grids, denoted as $N_{\text {over }}$, is counted by the number of grid points minus one. Thus, the total number of unknowns is $\left(2 N_{\text {over }}+2\right)$, and the grid size for the three-point finite surface is $\Delta x^{*}=2 / N_{\text {over }}$.

The discretized governing equation is applied to the three-point finite surface as well as the four-point finite surface, while the fourth-order Taylor-expansion equation is applied to the three-point finite surface. Moreover, the boundary condition is applied to the two $h^{\prime}$ unknown variables at the two ends. In total, we can derive $\left(2 N_{\text {over }}+2\right)$ independent algebraic equations. 
2.4.1 Three-point finite surface

Each three-point finite surface is associated with six unknown variables, $h_{p}^{*}, h_{p}^{*}, h_{1}^{*}, h_{1}^{*}, h_{2}^{*}$ and $h_{2}^{*}$. Their relationships are derived from the fourth-order Taylor expansion,

$$
\begin{gathered}
h_{1}^{*}=h_{p}^{*}-\frac{\Delta x^{*}}{2} h_{p}^{* \prime}+\frac{1}{2}\left(\frac{\Delta x^{*}}{2}\right)^{2} h_{p}^{* ”}-\frac{1}{3 !}\left(\frac{\Delta x^{*}}{2}\right)^{3} h_{p}^{*(3)}+\frac{1}{4 !}\left(\frac{\Delta x^{*}}{2}\right)^{4} h_{p}^{*(4)}-O\left(\Delta x^{* 5}\right), \\
h_{2}^{*}=h_{p}^{*}+\frac{\Delta x^{*}}{2} h_{p}^{* \prime}+\frac{1}{2}\left(\frac{\Delta x^{*}}{2}\right)^{2} h_{p}^{* \prime}+\frac{1}{3 !}\left(\frac{\Delta x^{*}}{2}\right)^{3} h_{p}^{*(3)}+\frac{1}{4 !}\left(\frac{\Delta x^{*}}{2}\right)^{4} h_{p}^{*(4)}+O\left(\Delta x^{* 5}\right), \\
h_{1}^{* \prime}=h_{p}^{*,}-\frac{\Delta x^{*}}{2} h_{p}^{* \prime}+\frac{1}{2}\left(\frac{\Delta x^{*}}{2}\right)^{2} h_{p}^{*(3)}-\frac{1}{3 !}\left(\frac{\Delta x^{*}}{2}\right)^{3} h_{p}^{*(4)}+O\left(\Delta x^{* 4}\right), \\
h_{2}^{*,}=h_{p}^{* \prime}+\frac{\Delta x^{*}}{2} h_{p}^{* \prime}+\frac{1}{2}\left(\frac{\Delta x^{*}}{2}\right)^{2} h_{p}^{*(3)}+\frac{1}{3 !}\left(\frac{\Delta x^{*}}{2}\right)^{3} h_{p}^{*(4)}+O\left(\Delta x^{* 4}\right) .
\end{gathered}
$$

By eliminating $h_{p}^{* \prime}, h_{p}^{*(3)}$ and $h_{p}^{*(4)}$ from the above four equations, we can derive the Taylor-expansion equation for the three-point finite surface,

$$
\begin{aligned}
h_{p}^{*^{\prime}}= & \left(6 h_{2}^{*}-6 h_{1}^{*}-\Delta x^{*} h_{1}^{* \prime}-\Delta x^{*} h_{2}^{* \prime}\right) / 4 \Delta x^{*}+O\left(\Delta x^{* 4}\right), \\
& \text { or }\left(h_{1}^{*{ }^{*}}+4 h_{p}^{*{ }^{*}}+h_{2}^{* \prime}\right) / 6-\left(h_{2}^{*}-h_{1}^{*}\right) / \Delta x^{*}=0,
\end{aligned}
$$

which involves the five unknown variables, $h_{p}^{* \prime}, h_{1}^{*}, h_{1}^{* \prime}, h_{2}^{*}$ and $h_{2}^{* \prime}$, and has the modelling error of $O\left(\Delta x^{* 4}\right)$.

Futhermore, the integral term in eqn (7) can be approximated by applying Simpson's rule with the three equally spaced grid points along the finite surface,

$$
\begin{aligned}
& \int_{x_{1}^{*}}^{x_{2}^{*}} f_{s}\left(x^{*}\right) \mathrm{d} x^{*}=\frac{\Delta x^{*}}{6}\left[f_{s}\left(x_{1}^{*}\right)+4 f_{s}\left(x_{p}^{*}\right)+f_{s}\left(x_{2}^{*}\right)\right]+O\left(\Delta x^{* 5}\right) \\
& =\frac{\Delta x^{*}}{6}\left(h_{1}^{*} \sqrt{1+h_{1}^{* 2}}+4 h_{p}^{*} \sqrt{1+h_{p}^{* 2}}+h_{2}^{*} \sqrt{1+h_{2}^{* 2}}\right)+O\left(\Delta x^{* 5}\right) .
\end{aligned}
$$

Substitute eqn (15) into eqn (7), and we can derive the discretized governing equation for the three-point finite surface,

$$
\frac{\Pi_{\sigma}}{6}\left(h_{1}^{*} \sqrt{1+h_{1}^{* \prime 2}}+4 h_{p}^{*} \sqrt{1+h_{p}^{* \prime 2}}+h_{2}^{*} \sqrt{1+h_{2}^{* \prime 2}}\right)-\frac{1}{\Delta x^{*}} \arctan \left(\frac{h_{2}^{* \prime}-h_{1}^{* \prime}}{1+h_{2}^{* \prime} h_{1}^{* \prime}}\right)=0
$$

which involves the six unknown variables, $h_{p}^{*}, h_{p}^{*}, h_{1}^{*}, h_{1}^{* \prime}, h_{2}^{*}$ and $h_{2}^{*}$, and has the modelling error of $O\left(\Delta x^{* 4}\right)$.

\subsubsection{Four-point finite surface}

The four-point finite surfaces are at the two ends of the overlapped grid, with a grid size of $3 \Delta x^{*} / 2$. They are associated with eight unknown variables, $h_{1}^{*}, h_{1}^{* \prime}, h_{1 p}^{*}, h_{1 p}^{* \prime}, h_{p 2}^{*} h_{p 2}^{*}, h_{2}^{*}$ and $h_{2}^{*}$, where the subscripts ' $1 \mathrm{p}$ ' and p2' indicate the equally spaced points between Point 1 and Point 2, from left to right, respectively. Thus, the integral term in eqn (7) can be approximated by applying the Simpson's 3/8 rule with the four grid points, 


$$
\begin{aligned}
& \int_{x_{1}^{*}}^{x_{2}^{*}} f_{s}\left(x^{*}\right) \mathrm{d} x^{*}=\frac{3 \Delta x^{*} / 2}{8}\left[f_{s}\left(x_{1}^{*}\right)+3 f_{s}\left(x_{1 p}^{*}\right)+3 f_{s}\left(x_{p 2}^{*}\right)+f_{s}\left(x_{2}^{*}\right)\right]+O\left(\Delta x^{* 5}\right) \\
= & \frac{3 \Delta x^{*}}{16}\left(h_{1}^{*} \sqrt{1+h_{1}^{* \prime 2}}+3 h_{1 p}^{*} \sqrt{1+h_{1 p}^{* \prime 2}}+3 h_{p 2}^{*} \sqrt{1+h_{p 2}^{* \prime 2}}+h_{2}^{*} \sqrt{1+h_{2}^{* 2}}\right)+O\left(\Delta x^{* 5}\right) .
\end{aligned}
$$

Substitute eqn (17) into eqn (7), and we can derive the discretized governing equation for the four-point finite surface,

$$
\frac{\Pi_{\sigma}}{8}\left(\begin{array}{l}
h_{1}^{*} \sqrt{1+h_{1}^{* \prime 2}}+3 h_{1 p}^{*} \sqrt{1+h_{1 p}^{* \prime 2}}+ \\
3 h_{p 2}^{*} \sqrt{1+h_{p 2}^{* \prime 2}}+h_{2}^{*} \sqrt{1+h_{2}^{* 22}}
\end{array}\right)-\frac{2}{3 \Delta x^{*}} \arctan \left(\frac{h_{2}^{* \prime}-h_{1}^{* \prime}}{1+h_{2}^{* \prime} h_{1}^{* \prime}}\right)=0,
$$

which involves the eight unknown variables, $h_{1}^{*}, h_{1}^{* \prime}, h_{1 p}^{*}, h_{1 p}^{* \prime}, h_{p 2}^{*} h_{p 2}^{* \prime}, h_{2}^{*}$ and $h_{2}^{* \prime}$, and has the modelling error of $O\left(\Delta x^{* 4}\right)$.

\subsection{Summary}

For the two grid layouts, we derive different discretized algebraic equations, as summarized in Table 1. From Table 1, we observe that the overlapped grid can achieve higher order of accuracy, $O\left(\Delta x^{* 4}\right)$, than that of the staggered grid, $O\left(\Delta x^{* 2}\right)$. Thus, presumably, we expect a better numerical performance with the overlapped grid.

We implement the FVM algorithm with MATLAB code, and adopt the Newton's method to iteratively find the converged solution. Also, we take advantage of the embedded mathematic library of MATLAB, such as the $\arctan ()$ function and the 64-bit Central Processing

\begin{tabular}{|c|c|c|c|c|}
\hline & & \multicolumn{2}{|c|}{ Overlapped Grid } & Staggered Grid \\
\hline \multicolumn{2}{|l|}{ Number of Grids } & \multicolumn{2}{|l|}{$N_{\text {over }}$} & $N_{\text {stag }}$ \\
\hline \multicolumn{2}{|c|}{ Number of Unknowns } & \multicolumn{2}{|l|}{$2 N_{\text {over }}+2$} & $2 N_{\text {stag }}+1$ \\
\hline \multicolumn{2}{|c|}{$\Delta x^{*}$} & \multicolumn{2}{|l|}{$2 / N_{\text {over }}$} & $1 / N_{\text {stag }}$ \\
\hline \multicolumn{2}{|c|}{ Number of Boundary Conditions } & \multicolumn{2}{|l|}{2} & 2 \\
\hline \multicolumn{2}{|c|}{$\begin{array}{l}\text { Governing Equation Algebraic } \\
\text { (Stress Balance) } \quad \text { Equation }\end{array}$} & $\begin{array}{l}\text { Eqn (16), for } \\
\text { three-point finite } \\
\text { surface }\end{array}$ & $\begin{array}{l}\text { Eqn }(18), \text { for } \\
\text { four-point finite } \\
\text { surface }\end{array}$ & Eqn (10) \\
\hline & Number & $N_{\text {over }}-1$ & 2 & $N_{\text {stag }}$ \\
\hline & Modelling error & $O\left(\Delta x^{* 4}\right)$ & $O\left(\Delta x^{* 4}\right)$ & $O\left(\Delta x^{* 2}\right)$ \\
\hline \multirow[t]{3}{*}{$\begin{array}{l}\text { Taylor-expansion } \\
\text { Equation }\end{array}$} & $\begin{array}{l}\text { Algebraic } \\
\text { Equation }\end{array}$ & \multicolumn{2}{|l|}{$\begin{array}{l}\text { Eqn (14), for } \\
\text { three-point finite } \\
\text { surface }\end{array}$} & Eqn (12) \\
\hline & Number & \multicolumn{2}{|l|}{$N_{\text {over }}-1$} & $N_{\text {stag }}-1$ \\
\hline & Modelling error & \multicolumn{2}{|l|}{$O\left(\Delta x^{* 4}\right)$} & $O\left(\Delta x^{* 2}\right)$ \\
\hline
\end{tabular}
Unit (CPU, i.e. a machine limit of $2.2204 \times 10^{-16}$ ), to approximate the Jacobian derivative

Table 1: Fact sheet of the two grid layouts. 


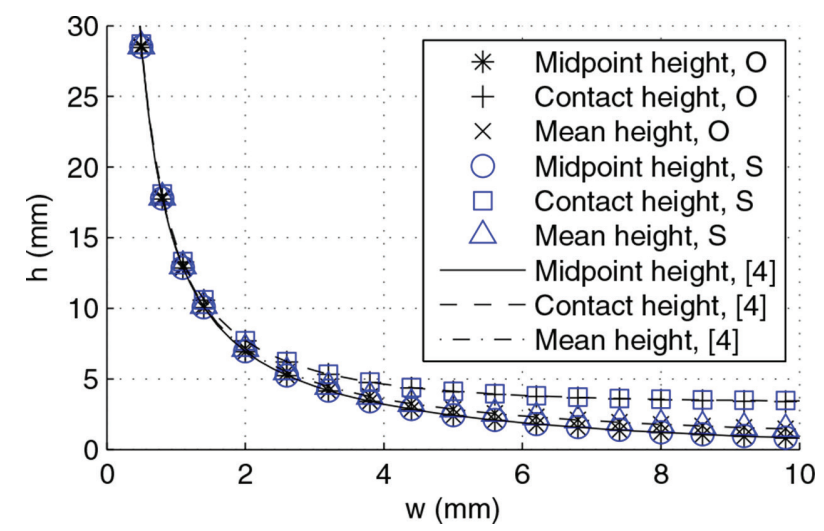

Figure 4: Comparison of the numerical solutions, with the published solution [4] by midpoint height, contact height and mean height. The grid numbers for both FVM solutions are 80. "O" stands for the overlapped grid, while "S" stands for the staggered grid.

matrix with a step size of $10^{-8}$ for each unknown variable. In this regard, the Parallel Computing Toolbox can accelerate the approximation of the Jacobian derivative matrix. The convergence criteria of the iterative solver is $10^{-13}$ for each algebraic equation. Generally, the computation completes within 6 iterations and the computational time is not a concern in the current study.

\section{RESULTS AND DISCUSSION}

\subsection{Verification with benchmark problem}

There is a recently published numerical solution [4] of the Young-Laplace equation by using iterated deferred corrections. The physical parameters are: $\rho=1000 \mathrm{~kg} / \mathrm{m}^{3}, g=9.8 \mathrm{~m} / \mathrm{s}^{2}$, $\sigma=72.2 \mathrm{~mJ} / \mathrm{m}^{2}$ and $\theta_{0}=\theta_{1}=14^{\circ}$. And, the dimensionless governing parameter is $\Pi_{\sigma}=1.36 \times 10^{5} w^{2}$. In this case, the two contact angles at the two plates are identical, and the free surface is symmetric about its midpoint.

In Fig. 4, we compare the three sets of numerical solutions by the midpoint height, the contact height and the mean height, with respect to the separation distance, $w$. The grid numbers for both FVM solutions are 80. Good agreement can be observed, which verifies the MATLAB solvers developed from the FVM algorithms. Note that the contact height (on the left end) is not directly defined for the staggered grid, as is shown in Fig. 3, and it is approximated by $h_{\text {contact }}^{*}=h_{\text {near }}^{*}-h_{\text {contact }}^{*} \Delta x^{*} / 2$, where $h_{\text {near }}^{*}$ represents the height at the neighboring grid point.

\subsection{Comparison between two layouts}

In this subsection, we compare the performance of the two grid layouts. The physical parameters are: $\rho=1000 \mathrm{~kg} / \mathrm{m}^{3}, g=9.8 \mathrm{~m} / \mathrm{s}^{2}, \sigma=72.2 \mathrm{~mJ} / \mathrm{m}^{2}, w=5 \mathrm{~mm}$ (i.e. $\Pi_{\sigma}=3.40$ ) and $\theta_{0}=\theta_{1}=28^{\circ}$. The grid numbers are doubled from 10 to 10240 (11 settings in total).

Figure 5 visualizes the numerical solutions from both grid layouts with 10 grids, 20 grids and 40 grids. It is observed that both grid layouts converge to the same solution. 
(a)

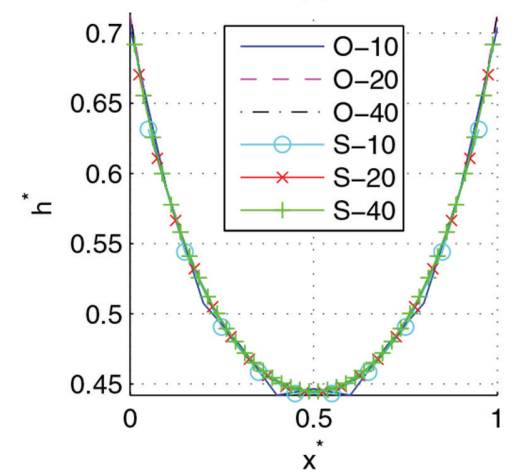

(b)

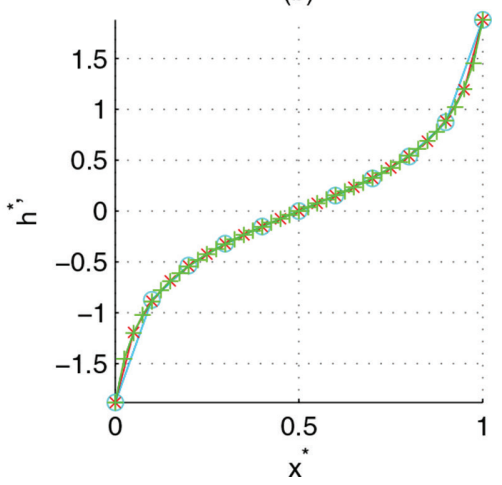

Figure 5: Numerical solutions with $\theta_{0}=\theta_{1}=28^{\circ}, \Pi_{\sigma}=3.40$, and $N=10,20$, or 40. (a) $h^{*}$; (b) $h^{* \prime}$.

Furthermore, Fig. 6 compares modelling errors of the two grid layouts, with respect to the total length of the free surface and $h^{*}$ and $h^{*}$ at three points. For the overlapped grid, the total length is defined as,

$$
L^{*}=\sum_{i=1}^{N_{\text {our }}} \sqrt{\left(x_{i}^{*}-x_{i+1}^{*}\right)^{2}+\left(h_{i}^{*}-h_{i+1}^{*}\right)^{2}}+O\left(\Delta x^{* 2}\right),
$$

where $x_{i}^{*}$ is the location associated with $h_{i}^{*}$. And, for the staggered grid, the total length is defined as,

$$
L^{*}=\sum_{i=1}^{N_{\text {saga }}-1} \sqrt{\left(x_{i}^{*}-x_{i+1}^{*}\right)^{2}+\left(h_{i}^{*}-h_{i+1}^{*}\right)^{2}}+\frac{\Delta x^{*}}{2}\left(\sqrt{1+h_{1}^{* \prime 2}}+\sqrt{1+h_{N_{s a q}+1}^{* \prime}{ }^{2}}\right)+O\left(\Delta x^{* 2}\right) .
$$

From Fig. 6a, it is observed that both grid layouts lead to the same converged total length of the free surface.

Moreover, since the grid number, $N$, is doubled consecutively, the Richardson extrapolation is applied to improve the modelling accuracy by exploiting the obtained numerical solutions. Then, based on the Richardson results, we define the modelling error as,

$$
\log _{10}(\text { error })=\log _{10}\left|1-X / X_{R}\right|,
$$

where $X$ denotes the numerical solution and $X_{R}$ denotes the corresponding $10^{\text {th }}$-order Richardson result. Thus, Fig. 6b-6d visualize those modelling errors for the total length, $h^{*}$ and $h^{* \prime}$ at three points.

From Fig. 6b, it is observed that both grid layouts can produce the total length with the modelling error in the order of $O\left(\Delta x^{* 2}\right)$. But, the overlapped grid has slightly better accuracy than the staggered grid, especially for $\log _{10}(N)>2$ or $N>100$. And, Fig. $6 \mathrm{c}$ and $\mathrm{d}$ visualize the modelling error of $h^{*}$ at $x^{*}=0,0.2$ and 0.4 and $h^{* *}$ at $x^{*}=0.2$ and 0.4. It is observed that the overlapped grid outperforms the staggered grid in terms of the modelling error by two orders for sufficient $N$, as is expected in Section 2.5. 

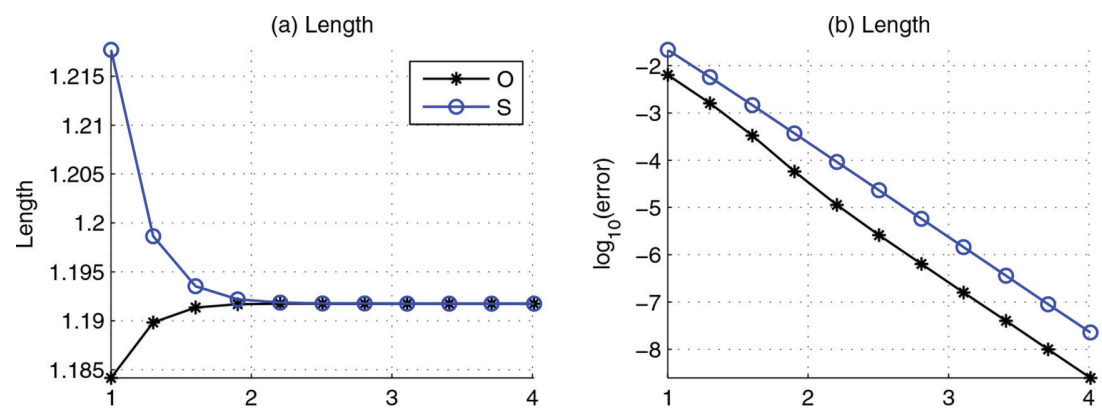

(c) $\mathrm{h}^{*}$

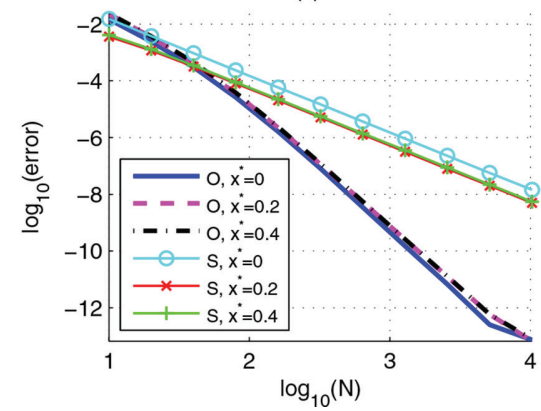

(d) $\mathrm{h}^{*}$

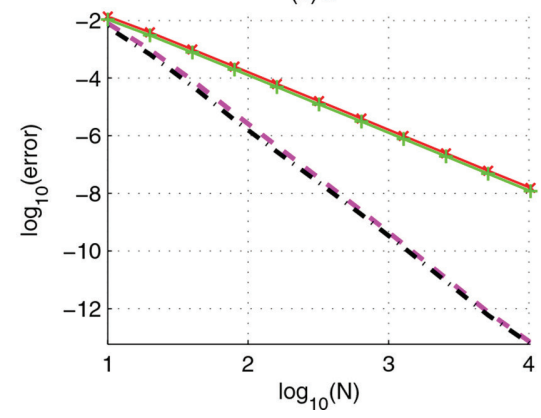

Figure 6: Numerical solutions with $\theta_{0}=\theta_{1}=28^{\circ}, \Pi_{\sigma}=3.40$, against different grid numbers. (a) total length of the free surface; (b) modelling errors of the total length; (c) modelling errors of $h^{*}$ at $x^{*}=0,0.2$, and 0.4 ; (d) modelling errors of $h^{* \prime}$ at $x^{*}=0.2$ and 0.4 .

By overlapping one more layer of grids on the staggered grid, the overlapped grid heavily packs more unknown variables for the finite surface. As a result, the overlapped grid demonstrates two major advantages:

1. the nonlinear source term is approximated with a higher order of accuracy, while the fourth order Taylor-expansion equation also has higher order of accuracy. Thus, as analyzed in Section 2.5, the overlapped grid can achieve a higher order of accuracy, $O\left(\Delta x^{* 4}\right)$, than the staggered grid, $O\left(\Delta x^{* 2}\right)$ for a sufficient grid number. Note that a similar order of accuracy can be achieved by adopting the quadratic interpolation [24] or even higherorder schemes [21] for the staggered grid, which potentionally suffers from the numerical wiggling and calls for artificial diffusion fragment [22].

2. Due to the multi-variables defined at one grid point, the effort is minimized to implement various boundary conditions, such as the Dirichlet boundary condition, the Neumann boundary condition or the Robin boundary condition. Also, the full states defined at one grid point offers convenience to multi-physics problems, where the free surface is coupled with other physical processes or between two fluid phases.

\section{CONCLUDING REMARKS}

In the current work, we propose and demonstrate an FVM with the overlapped grid to numerically solve the Young-Laplace equation and achieve a higher order of accuracy, $O\left(\Delta x^{4}\right)$, 
compared with the "traditional" staggered grid, $O\left(\Delta x^{2}\right)$. Mainly, the improved accuracy results from the heavily packed unknown variables and the better approximation of the nonlinear source term. Also, the multi-variables defined at each grid point offer convenience to the implementation of the boundary conditions and the coupling with multi-physics, especially for two-phase flow problems.

\section{ACKNOWLEDGMENT}

The financial support from the Research Grants Council of Hong Kong (GRF17237316, GRF 17211115, GRF17207914, GRF HKU717613E, and GRF HKU718111E) and the University of Hong Kong (URC 201511159108, 201411159074 and 201311159187) is gratefully acknowledged. The work is also supported in part by the Zhejiang Provincial, Hangzhou Municipal, and Lin'an County Governments. The first author also acknowledges the Hong Kong Ph.D. Fellowship Scheme funded by the Hong Kong Research Grants Council (RGC).

\section{REFERENCES}

[1] Shaughnessy, E.J., Introduction to Fluid Mechanics, Oxford University Press: New York \& Oxford, 2010.

[2] Quere, D., Wetting and roughness. Annual Review of Materials Research, 38, pp. 71-99, 2008. https://doi.org/10.1146/annurev.matsci.38.060407.132434

[3] Bashforth, F. \& Adams, J.C., An Attempt to Test the Theory of Capillary Action, Cambridge University Press: Warehouse, London, 1883.

[4] Bullard, J.W. \& Garboczi, E.J., Capillary rise between planar surfaces. Physical Review E, 79(011604), pp. 1-8, 2009.

https://doi.org/10.1103/physreve.79.011604

[5] Roura, P., Contact angle in thick capillaries: a derivation based on energy balance. European Journal of Physics, 28(4), pp. 27-32, 2007.

https://doi.org/10.1088/0143-0807/28/4/L01

[6] Zhmud, B.V., Tiberg, F. \& Hallstensson, K., Dynamics of capillary rise. Journal of Colloid and Interface Science, 228(2), pp. 263-269, 2000.

https://doi.org/10.1006/jcis.2000.6951

[7] Taroni, M. \& Vella, D., Multiple equilibria in a simple elastocapillary system. Journal of Fluid Mechanics, 712, pp. 273-294, 2012.

https://doi.org/10.1017/jfm.2012.418

[8] Weber, M.W. \& Shandas, R., Computational fluid dynamics analysis of microbubble formation in microfluidic flow-focusing devices. Microfluidics and Nanofluidics, 3(2), pp. 195-206, 2007.

https://doi.org/10.1007/s10404-006-0120-9

[9] Dadvand, A., Khoo, B.C., Shervani-Tabar, M.T. \& Khalilpourazary, S., Boundary element analysis of the droplet dynamics induced by spark-generated bubble. Engineering Analysis with Boundary Elements, 36(11), pp. 1595-1603, 2012. https://doi.org/10.1016/j.enganabound.2012.04.009

[10] Lim, L.K., Hua, J.S., Wang, C.H. \& Smith, K.A., Numerical simulation of cone-jet formation in electrohydrodynamic atomization. AIChE Journal, 57(1), pp. 57-78, 2011. https://doi.org/10.1002/aic.12254 
[11] Sprittles, J.E. \& Shikhmurzaev, Y.D., Finite element simulation of dynamic wetting flows as an interface formation process. Journal of Computational Physics, 233, pp. 34-65, 2013. https://doi.org/10.1016/j.jcp.2012.07.018

[12] Sprittles, J.E. \& Shikhmurzaev, Y.D., Finite element framework for describing dynamic wetting phenomena. International Journal for Numerical Methods in Fluids, 68(10), pp. 1257-1298, 2012. https://doi.org/10.1002/fld.2603

[13] Rayleigh, L., On the instability of jets. Proceedings of the London Mathematical Society, 1(1), pp. 4-13, 1878. https://doi.org/10.1112/plms/s1-10.1.4

[14] Batchelor, G.K., The stability of a large gas bubble rising through liquid. Journal of Fluid Mechanics, 184, pp. 399-422, 1987. https://doi.org/10.1017/S0022112087002945

[15] Sussman, M. \& Smereka, P., Axisymmetric free boundary problems. Journal of Fluid Mechanics, 341, pp. 269-294, 1997. https://doi.org/10.1017/S0022112097005570

[16] Berry, J.D., Neeson, M.J., Dagastine, R.R., Chan, D.Y.C. \& Tabor, R.F., Measurement of surface and interfacial tension using pendant drop tensiometry. Journal of Colloid Interface Science, 454, pp. 226-237, 2015. https://doi.org/10.1016/j.jcis.2015.05.012

[17] Rio, O.I. \& Neumann, A.W., Axisymmetric drop shape analysis: computational methods for the measurement of interfacial properties from the shape and dimensions of pendant and sessile drops. Journal of Colloid Interface Science, 196(2), pp. 136-147, 1997. https://doi.org/10.1006/jcis.1997.5214

[18] Cabezas, M.G., Bateni, A., Montanero, J.M. \& Neumann, A.W., Determination of surface tension and contact angle from the shapes of axisymmetric fluid interfaces without use of apex coordinates. Langmuir, 22(24), pp. 10053-10060, 2006. https://doi.org/10.1021/la061928t

[19] Dingle, N.M., Tjiptowidjojo, K., Basaran, O.A. \& Harris, M.T., A finite element based algorithm for determining interfacial tension (gamma) from pendant drop profiles. Journal of Colloid Interface Science, 286(2), pp. 647-660, 2005. https://doi.org/10.1016/j.jcis.2005.01.052

[20] Gille, M., Gorbacheva, Y., Hahn, A., Polevikov, V. \& Tobiska, L., Simulation of a pending drop at a capillary tip. Communications in Nonlinear Science and Numerical Simulation, 26(1-3), pp. 137-151, 2015. https://doi.org/10.1016/j.cnsns.2015.02.007

[21] Danov, K.D., Dimova, S.N., Ivanov, T.B. \& Novev, J.K., Shape analysis of a rotating axisymmetric drop in gravitational field: Comparison of numerical schemes for real-time data processing. Colloids and Surfaces a-Physicochemical and Engineering Aspects, 489, pp. 75-85, 2016.

[22] Versteeg, H.K. \& Malalasekera, W., An Introduction to Computational Fluid Dynamics: the Finite Volume Method, Pearson Education, Harlow, 2007.

[23] Patankar, S.V., Numerical Heat Transfer and Fluid Flow, CRC Press, New York, 1980.

[24] Leonard, B.P., Order of accuracy of quick and related convection-diffusion Schemes. Applied Mathematical Modelling, 19(11), pp. 640-653, 1995.

https://doi.org/10.1016/0307-904X(95)00084-W 